\title{
I'm a Belieber: Social Roles via Self-identification and Conceptual Attributes
}

\author{
Charley Beller, Rebecca Knowles, Craig Harman \\ Shane Bergsma ${ }^{\dagger}$, Margaret Mitchell ${ }^{\ddagger}$, Benjamin Van Durme \\ Human Language Technology Center of Excellence \\ Johns Hopkins University, Baltimore, MD USA \\ ${ }^{\dagger}$ University of Saskatchewan, Saskatoon, Saskatchewan Canada \\ ${ }^{\ddagger}$ Microsoft Research, Redmond, Washington USA \\ charleybeller@jhu.edu,rknowles@jhu.edu, craig@craigharman.net, \\ shane.a.bergsma@gmail.com,memitc@microsoft.com, vandurme@cs.jhu.edu
}

\begin{abstract}
Motivated by work predicting coarsegrained author categories in social media, such as gender or political preference, we explore whether Twitter contains information to support the prediction of finegrained categories, or social roles. We find that the simple self-identification pattern "I am a_" supports significantly richer classification than previously explored, successfully retrieving a variety of fine-grained roles. For a given role (e.g., writer), we can further identify characteristic attributes using a simple possessive construction (e.g., writer's _ ). Tweets that incorporate the attribute terms in first person possessives (my __ ) are confirmed to be an indicator that the author holds the associated social role.
\end{abstract}

\section{Introduction}

With the rise of social media, researchers have sought to induce models for predicting latent $a u$ thor attributes such as gender, age, and political preferences (Garera and Yarowsky, 2009; Rao et al., 2010; Burger et al., 2011; Van Durme, 2012b; Zamal et al., 2012). Such models are clearly in line with the goals of both computational advertising (Wortman, 2008) and the growing area of computational social science (Conover et al., 2011; Nguyen et al., 2011; Paul and Dredze, 2011; Pennacchiotti and Popescu, 2011; Mohammad et al., 2013) where big data and computation supplement methods based on, e.g., direct human surveys. For example, Eisenstein et al. (2010) demonstrated a model that predicted where an author was located in order to analyze regional distinctions in communication. While some users explicitly share their GPS coordinates through their
Twitter clients, having a larger collection of automatically identified users within a region was preferable even though the predictions for any given user were uncertain.

We show that media such as Twitter can support classification that is more fine-grained than gender or general location. Predicting social roles such as doctor, teacher, vegetarian, christian, may open the door to large-scale passive surveys of public discourse that dwarf what has been previously available to social scientists. For example, work on tracking the spread of flu infections across Twitter (Lamb et al., 2013) might be enhanced with a factor based on aggregate predictions of author occupation.

We present two studies showing that firstperson social content (tweets) contains intuitive signals for such fine-grained roles. We argue that non-trivial classifiers may be constructed based purely on leveraging simple linguistic patterns. These baselines suggest a wide range of author categories to be explored further in future work.

Study 1 In the first study, we seek to determine whether such a signal exists in self-identification: we rely on variants of a single pattern, "I am a _.", to bootstrap data for training balanced-class binary classifiers using unigrams observed in tweet content. As compared to prior research that required actively polling users for ground truth in order to construct predictive models for demographic information (Kosinski et al., 2013), we demonstrate that some users specify such properties publicly through direct natural language.

Many of the resultant models show intuitive strongly-weighted features, such as a writer being likely to tweet about a story, or an athlete discussing a game. This demonstrates selfidentification as a viable signal in building predictive models of social roles. 


\begin{tabular}{ll}
\hline \hline Role & Tweet \\
\hline artist & I'm an Artist..... the last of a dying breed \\
\hline belieber & $\begin{array}{l}\text { @ justinbieber I will support you in ev- } \\
\text { erything you do because I am a belieber } \\
\text { please follow me I love you 30 }\end{array}$ \\
\hline vegetarian & So glad I'm a vegetarian.
\end{tabular}

Table 1: Examples of self-identifying tweets.

\begin{tabular}{cc|cc|cc}
\hline \hline$\#$ & Role & $\#$ & Role & $\#$ & Role \\
\hline 29,924 & little & 5,694 & man & 564 & champion \\
21,822 & big & $\ldots$ & $\ldots$ & 559 & teacher \\
18,957 & good & 4,007 & belieber & 556 & writer \\
13,069 & huge & 3,997 & celebrity & 556 & awful \\
13,020 & bit & 3,737 & virgin & $\ldots$ & $\ldots$ \\
12,816 & fan & 3,682 & pretty & 100 & cashier \\
10,832 & bad & $\ldots$ & $\ldots$ & 100 & bro \\
10,604 & girl & 2,915 & woman & $\ldots$ & $\ldots$ \\
9,981 & very & 2,851 & beast & 10 & linguist \\
$\ldots$ & $\ldots$ & $\ldots$ & $\ldots$ & $\ldots$ & $\ldots$
\end{tabular}

Table 2: Number of self-identifying users per "role". While rich in interesting labels, cases such as very highlight the purposeful simplicity of the current approach.

Study 2 In the second study we exploit a complementary signal based on characteristic conceptual attributes of a social role, or concept class (Schubert, 2002; Almuhareb and Poesio, 2004; Paşca and Van Durme, 2008). We identify typical attributes of a given social role by collecting terms in the Google n-gram corpus that occur frequently in a possessive construction with that role. For example, with the role doctor we extract terms matching the simple pattern "doctor's _".

\section{Self-identification}

All role-representative users were drawn from the free public $1 \%$ sample of the Twitter Firehose, over the period 2011-2013, from the subset that selected English as their native language $(85,387,204$ unique users). To identify users of a particular role, we performed a case-agnostic search of variants of a single pattern: I am $a(n)$ $\ldots$, and I'm $a(n) \ldots$, where all single tokens filling the slot were taken as evidence of the author selfreporting for the given "role". Example tweets can be seen in Table 1, examples of frequency per role in Table 2. This resulted in 63,858 unique roles identified, of which 44,260 appeared only once. ${ }^{1}$

We manually selected a set of roles for further exploration, aiming for a diverse sample across: occupation (e.g., doctor, teacher), family (mother), disposition (pessimist), religion (chris-

\footnotetext{
${ }^{1}$ Future work should consider identifying multi-word role labels (e.g., Doctor Who fan, or dog walker).
}

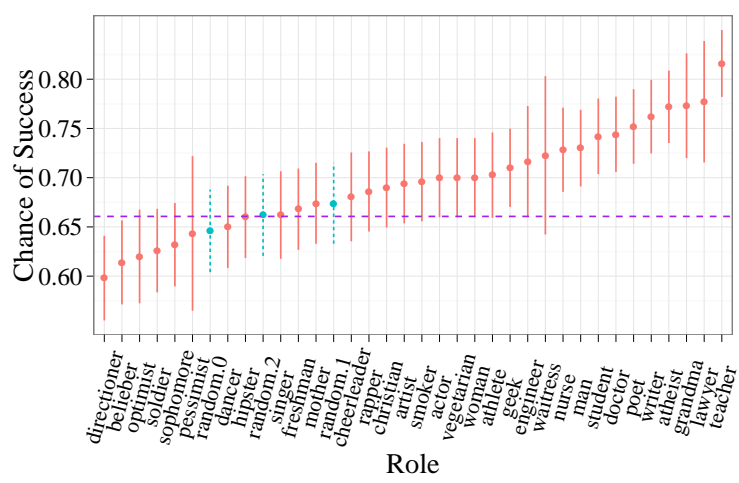

Figure 1: Success rate for querying a user. Random.0,1,2 are background draws from the population, with the mean of those three samples drawn horizontally. Tails capture $95 \%$ confidence intervals

tian), and "followers" (belieber, directioner). ${ }^{2}$ We filtered users via language ID (Bergsma et al., 2012) to better ensure English content. ${ }^{3}$

For each selected role, we randomly sampled up to 500 unique self-reporting users and then queried Twitter for up to 200 of their recent publicly posted tweets. ${ }^{4}$ These tweets served as representative content for that role, with any tweet matching the self-reporting patterns filtered. Three sets of background populations were extracted based on randomly sampling users that self-reported English (post-filtered via LID).

Twitter users are empowered to at any time delete, rename or make private their accounts. Any given user taken to be representative based on a previously posted tweet may no longer be available to query on. As a hint of the sort of user studies one might explore given access to social role prediction, we see in Figure 1 a correlation between self-reported role and the chance of an account still being publicly visible, with roles such as belieber and directioner on the one hand, and doctor and teacher on the other.

The authors examined the self-identifying tweet of 20 random users per role. The accuracy of the self-identification pattern varied across roles and is attributable to various factors including quotes, e.g. @StarTrek Jim, I'm a DOCTOR not a download!. While these samples are small (and thus estimates of quality come with wide variance), it

\footnotetext{
${ }^{2}$ Those that follow the music/life of the singer Justin Bieber and the band One Direction, respectively.

${ }^{3}$ This removes users that selected English as their primary language, used a self-identification phrase, e.g. I am a belieber, but otherwise tended to communicate in non-English.

${ }^{4}$ Roughly half of the classes had less than 500 selfreporting users in total, in those cases we used all matches.
} 


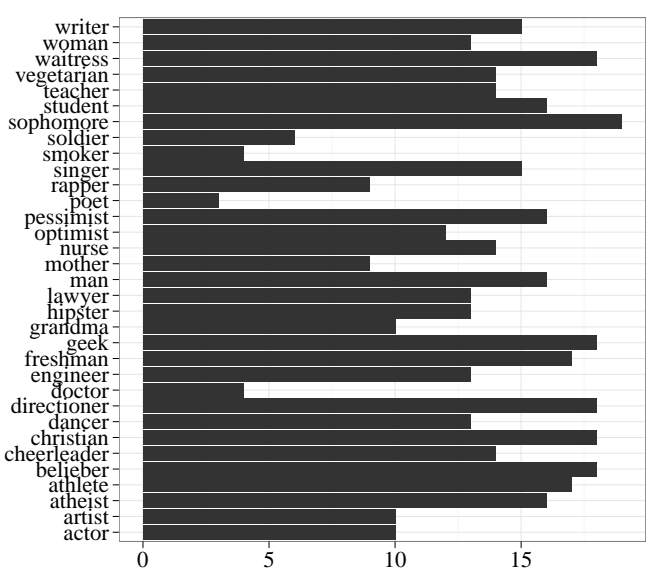

Figure 2: Valid self-identifying tweets from sample of 20.

is noteworthy that a non-trivial number for each were judged as actually self-identifying.

Indicative Language Most work in user classification relies on featurizing language use, most simply through binary indicators recording whether a user did or did not use a particular word in a history of $n$ tweets. To explore whether language provides signal for future work in fine-grain social role prediction, we constructed a set of experiments, one per role, where training and test sets were balanced between users from a random background sample and self-reported users. Baseline accuracy in these experiments was thus $50 \%$.

Each training set had a target of 600 users (300 background, 300 self-identified); for those roles with less than 300 users self-identifying, all users were used, with an equal number background. We used the Jerboa (Van Durme, 2012a) platform to convert data to binary feature vectors over a unigram vocabulary filtered such that the minimum frequency was 5 (across unique users). Training and testing was done with a log-linear model via LibLinear (Fan et al., 2008). We used the positively annotated data to form test sets, balanced with data from the background set. Each test set had a theoretical maximum size of 40 , but for several classes it was in the single digits (see Figure 2). Despite the varied noisiness of our simple pattern-bootstrapped training data, and the small size of our annotated test set, we see in Figure 3 that we are able to successfully achieve statistically significant predictions of social role for the majority of our selected examples.

Table 3 highlights examples of language indicative of role, as determined by the most positively weighted unigrams in the classification experi-

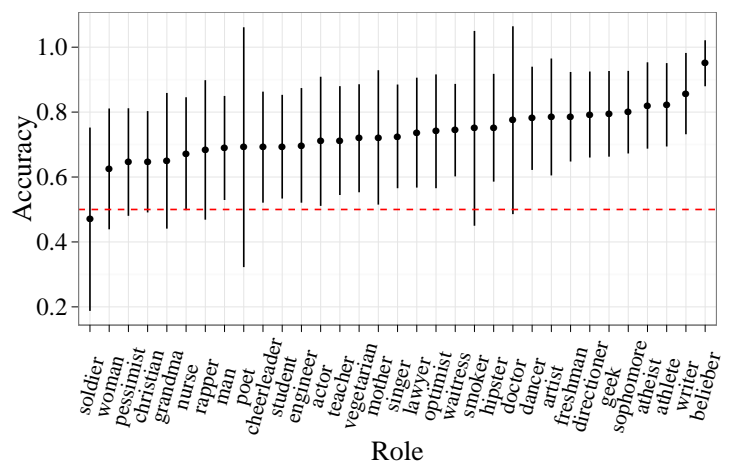

Figure 3: Accuracy in classifying social roles.

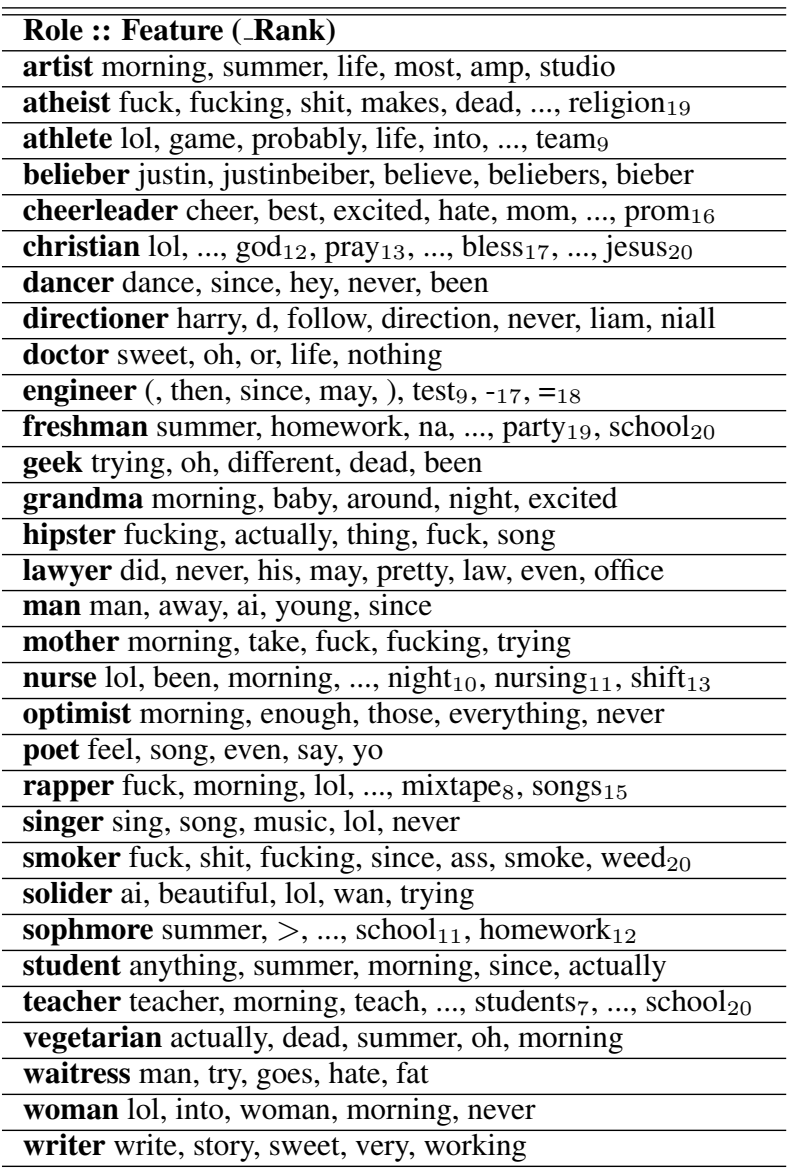

Table 3: Most-positively weighted features per role, along with select features within the top 20 . Surprising mother features come from ambigious self-identification, as seen in tweets such as: I'm a motherf!'cking starrrrr.

ment. These results qualitatively suggest many roles under consideration may be teased out from a background population by focussing on language that follows expected use patterns. For example the use of the term game by athletes, studio by artists, mixtape by rappers, or jesus by Christians.

\section{Characteristic Attributes}

Bergsma and Van Durme (2013) showed that the 
task of mining attributes for conceptual classes can relate straightforwardly to author attribute prediction. If one views a role, in their case gender, as two conceptual classes, male and female, then existing attribute extraction methods for third-person content (e.g., news articles) can be cheaply used to create a set of bootstrapping features for building classifiers over first-person content (e.g., tweets). For example, if we learn from news corpora that: a man may have a wife, then a tweet saying: ...my wife... can be taken as potential evidence of membership in the male conceptual class.

In our second study, we test whether this idea extends to our wider set of fine-grained roles. For example, we aimed to discover that a doctor may have a patient, while a hairdresser may have a salon; these properties can be expressed in firstperson content as possessives like my patient or my salon. We approached this task by selecting target roles from the first experiment and ranking characteristic attributes for each using pointwise mutual information (PMI) (Church and Hanks, 1990).

First, we counted all terms matching a target social role's possessive pattern (e.g., doctor's _ ) in the web-scale n-gram corpus Google V2 (Lin et al., 2010) ${ }^{5}$. We ranked the collected terms by computing PMI between classes and attribute terms. Probabilities were estimated from counts of the class-attribute pairs along with counts matching the generic possessive patterns his _ and her _ which serve as general background categories. Following suggestions by Bergsma and Van Durme, we manually filtered the ranked list. ${ }^{6}$ We removed attributes that were either (a) not nominal, or (b) not indicative of the social role. This left fewer than 30 attribute terms per role, with many roles having fewer than 10 .

We next performed a precision test to identify potentially useful attributes in these lists. We examined tweets with a first person possessive pattern for each attribute term from a small corpus of tweets collected over a single month in 2013, discarding those attribute terms with no positive matches. This precision test is useful regardless of how attribute lists are generated. The attribute

\footnotetext{
${ }^{5}$ In this corpus, follower-type roles like belieber and directioner are not at all prevalent. We therefore focused on occupational and habitual roles (e.g., doctor, smoker).

${ }^{6}$ Evidence from cognitive work on memory-dependent tasks suggests that such relevance based filtering (recognition) involves less cognitive effort than generating relevant attributes (recall) see (Jacoby et al., 1979). Indeed, this filtering step generally took less than a minute per class.
}

term chart, for example, had high PMI with doctor; but a precision test on the phrase my chart yielded a single tweet which referred not to a medical chart but to a top ten list (prompting removal of this attribute). Using this smaller high-precision set of attribute terms, we collected tweets from the Twitter Firehose over the period 2011-2013.

\section{Attribute-based Classification}

Attribute terms are less indicative overall than self-ID, e.g., the phrase I'm a barber is a clearer signal than my scissors. We therefore include a role verification step in curating a collection of positively identified users. We use the crowdsourcing platform Mechanical Turk ${ }^{7}$ to judge whether the person tweeting held a given role Tweets were judged 5-way redundantly. Mechanical Turk judges ("Turkers") were presented with a tweet and the prompt: Based on this tweet, would you think this person is a BARBER/HAIRDRESSER? along with four response options: Yes, Maybe, Hard to tell, and No.

We piloted this labeling task on 10 tweets per attribute term over a variety of classes. Each answer was associated with a score (Yes $=1$, Maybe $=.5$, Hard to tell $=\mathrm{No}=0$ ) and aggregated across the five judges. We found in development that an aggregate score of 4.0 (out of 5.0) led to an acceptable agreement rate between the Turkers and the experimenters, when the tweets were randomly sampled and judged internally. We found that making conceptual class assignments based on a single tweet was often a subtle task. The results of this labeling study are shown in Figure 4, which gives the percent of tweets per attribute that were 4.0 or above. Attribute terms shown in red were manually discarded as being inaccurate (low on the y-axis) or non-prevalent (small shape).

From the remaining attribute terms, we identified users with tweets scoring 4.0 or better as positive examples of the associated roles. Tweets from those users were scraped via the Twitter API to construct corpora for each role. These were split intro train and test, balanced with data from the same background set used in the self-ID study.

Test sets were usually of size 40 ( 20 positive, 20 background), with a few classes being sparse (the smallest had only 16 instances). Results are shown in Figure 5. Several classes in this balanced setup can be predicted with accuracies in the 70-90\%

\footnotetext{
${ }^{7}$ https://www.mturk.com/mturk/
} 

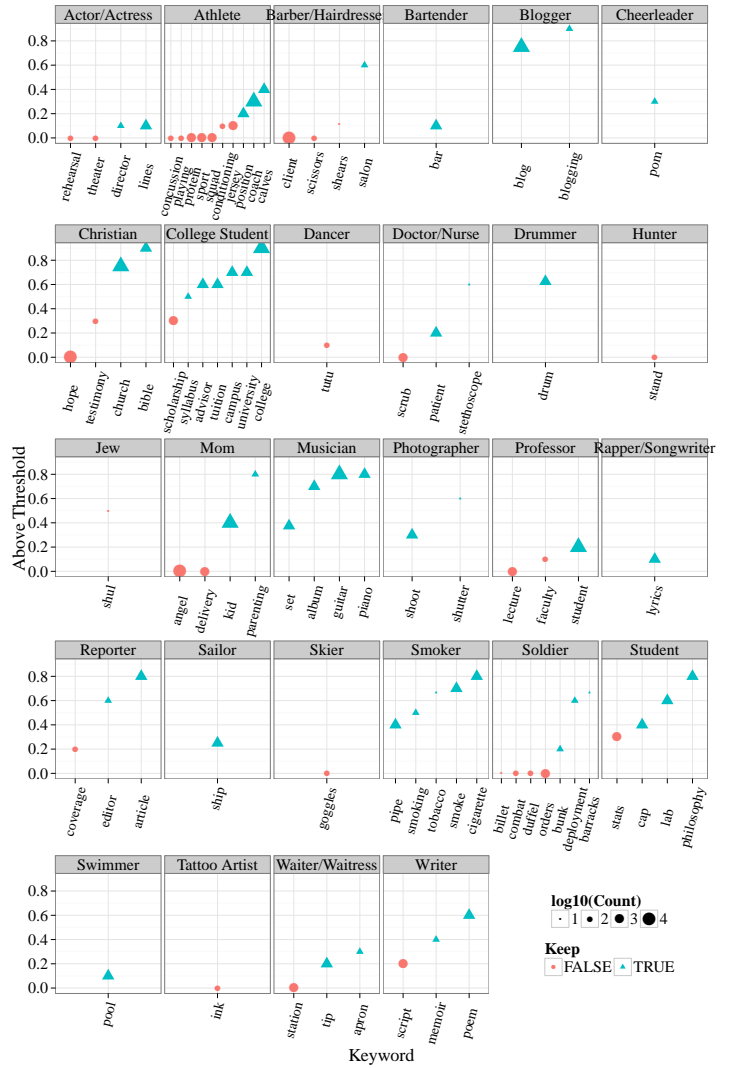

Figure 4: Turker judged quality of attributes selected as candidate features for bootstrapping positive instances of the given social role.

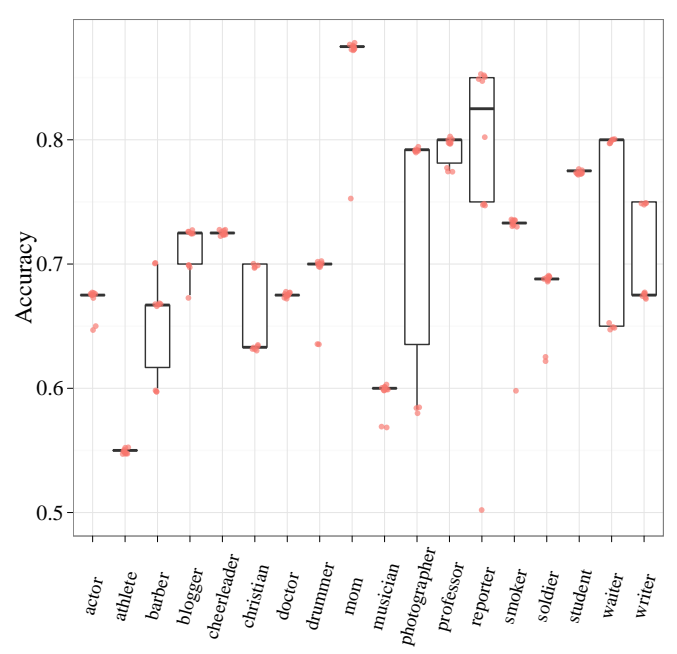

Figure 5: Classifier accuracy on balanced set contrasting agreed upon Twitter users of a given role against users pulled at random from the $1 \%$ stream.

range, supporting our claim that there is discriminating content for a variety of these social roles.

Conditional Classification How accurately we can predict membership in a given class when a Twitter user sends a tweet matching one of the targeted attributes? For example, if one sends a tweet saying my coach, then how likely is it that author

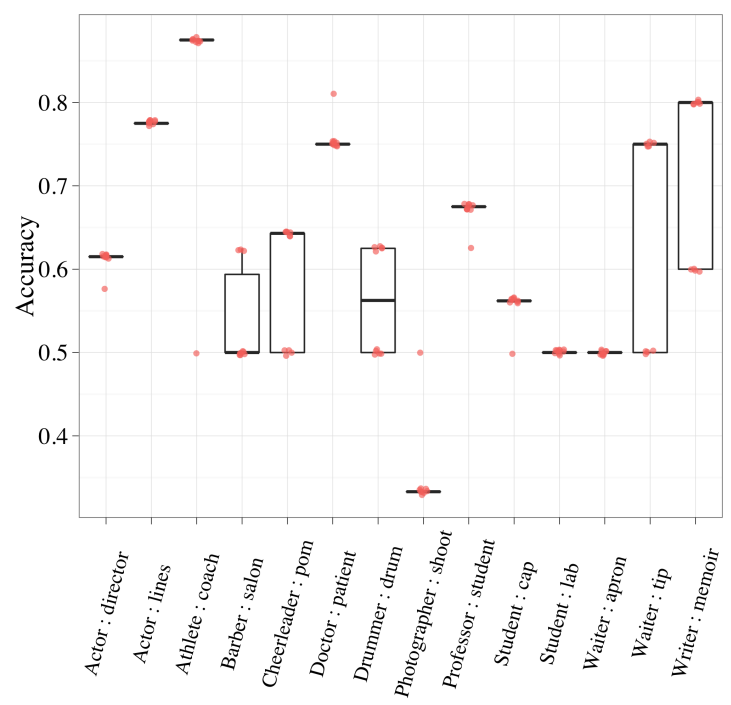

Figure 6: Results of positive vs negative by attribute term. Given that a user tweets ... my lines ... we are nearly $80 \%$ accurate in identifying whether or not the user is an actor.

\section{is an athlete?}

Using the same collection as the previous experiment, we trained classifiers conditioned on a given attribute term. Positive instances were taken to be those with a score of 4.0 or higher, with negative instances taken to be those with scores of 1.0 or lower (strong agreement by judges that the original tweet did not provide evidence of the given role). Classification results are shown in Figure 6.

\section{Conclusion}

We have shown that Twitter contains sufficiently robust signal to support more fine-grained author attribute prediction tasks than have previously been attempted. Our results are based on simple, intuitive search patterns with minimal additional filtering: this establishes the feasibility of the task, but leaves wide room for future work, both in the sophistication in methodology as well as the diversity of roles to be targeted. We exploited two complementary types of indicators: self-identification and self-possession of conceptual class (role) attributes. Those interested in identifying latent demographics can extend and improve these indicators in developing ways to identify groups of interest within the general population of Twitter users.

Acknowledgements This material is partially based on research sponsored by the NSF under grants DGE-123285 and IIS-1249516 and by DARPA under agreement number FA8750-13-20017 (the DEFT program). 


\section{References}

Abdulrahman Almuhareb and Massimo Poesio. 2004. Attribute-based and value-based clustering: an evaluation. In Proceedings of EMNLP.

Shane Bergsma and Benjamin Van Durme. 2013. Using Conceptual Class Attributes to Characterize Social Media Users. In Proceedings of ACL.

Shane Bergsma, Paul McNamee, Mossaab Bagdouri, Clay Fink, and Theresa Wilson. 2012. Language identification for creating language-specific twitter collections. In Proceedings of the NAACL Workshop on Language and Social Media.

John D. Burger, John Henderson, George Kim, and Guido Zarrella. 2011. Discriminating gender on twitter. In Proceedings of EMNLP.

Kenneth Ward Church and Patrick Hanks. 1990. Word association norms, mutual information, and lexicography. Computational linguistics, 16(1):22-29.

Michael Conover, Jacob Ratkiewicz, Matthew Francisco, Bruno Gonçalves, Filippo Menczer, and Alessandro Flammini. 2011. Political polarization on twitter. In ICWSM.

Jacob Eisenstein, Brendan O'Connor, Noah Smith, and Eric P. Xing. 2010. A latent variable model of geographical lexical variation. In Proceedings of EMNLP

Rong-En Fan, Kai-Wei Chang, Cho-Jui Hsief, XiangRui Wang, and Chih-Jen Lin. 2008. Liblinear: A library for large linear classification. Journal of Machine Learning Research, (9).

Nikesh Garera and David Yarowsky. 2009. Modeling latent biographic attributes in conversational genres. In Proceedings of ACL.

Larry L Jacoby, Fergus IM Craik, and Ian Begg. 1979. Effects of decision difficulty on recognition and recall. Journal of Verbal Learning and Verbal Behavior, 18(5):585-600.

Michal Kosinski, David Stillwell, and Thore Graepel. 2013. Private traits and attributes are predictable from digital records of human behavior. Proceedings of the National Academy of Sciences.

Alex Lamb, Michael J. Paul, and Mark Dredze. 2013. Separating fact from fear: Tracking flu infections on twitter. In Proceedings of NAACL.

Dekang Lin, Kenneth Church, Heng Ji, Satoshi Sekine, David Yarowsky, Shane Bergsma, Kailash Patil, Emily Pitler, Rachel Lathbury, Vikram Rao, Kapil Dalwani, and Sushant Narsale. 2010. New tools for web-scale n-grams. In Proc. LREC, pages 22212227.
Saif M. Mohammad, Svetlana Kiritchenko, and Joel Martin. 2013. Identifying purpose behind electoral tweets. In Proceedings of the Second International Workshop on Issues of Sentiment Discovery and Opinion Mining, WISDOM '13, pages 1-9.

Dong Nguyen, Noah A Smith, and Carolyn P Rosé. 2011. Author age prediction from text using linear regression. In Proceedings of the 5th ACLHLT Workshop on Language Technology for Cultural Heritage, Social Sciences, and Humanities, pages 115-123. Association for Computational Linguistics.

Marius Paşca and Benjamin Van Durme. 2008. Weakly-Supervised Acquisition of Open-Domain Classes and Class Attributes from Web Documents and Query Logs. In Proceedings of ACL.

Michael J Paul and Mark Dredze. 2011. You are what you tweet: Analyzing twitter for public health. In ICWSM.

Marco Pennacchiotti and Ana-Maria Popescu. 2011. Democrats, Republicans and Starbucks afficionados: User classification in Twitter. In Proceedings of the 17th ACM SIGKDD International Conference on Knowledge Discovery and Data mining, pages 430438. ACM.

Delip Rao, David Yarowsky, Abhishek Shreevats, and Manaswi Gupta. 2010. Classifying latent user attributes in twitter. In Proceedings of the Workshop on Search and Mining User-generated Contents (SMUC).

Lenhart K. Schubert. 2002. Can we derive general world knowledge from texts? In Proceedings of HLT.

Benjamin Van Durme. 2012a. Jerboa: A toolkit for randomized and streaming algorithms. Technical Report 7, Human Language Technology Center of Excellence, Johns Hopkins University.

Benjamin Van Durme. 2012b. Streaming analysis of discourse participants. In Proceedings of EMNLP.

Jennifer Wortman. 2008. Viral marketing and the diffusion of trends on social networks. Technical Report MS-CIS-08-19, University of Pennsylvania, May.

Faiyaz Al Zamal, Wendy Liu, and Derek Ruths. 2012. Homophily and latent attribute inference: Inferring latent attributes of Twitter users from neighbors. In Proceedings of ICWSM. 